\title{
Varicella myocarditis presenting with unusual ventricular arrhythmias
}

\author{
G. I. FIDDLER 1 , R. W. F. CAMPBELL ${ }^{2}$, A. POTTAGE, \\ AND M. J. GODMAN \\ From the Department of Cardiology, Royal Hospital for Sick Children, and the Departments of \\ Cardiology and Therapeutics, Royal Infirmary, Edinburgh
}

\begin{abstract}
A 10-year-old boy with varicella developed syncopal episodes caused by runs of self-terminating ventricular tachycardia and ventricular fibrillation. These arrhythmias were thought to be caused by varicella myocarditis and were resistant to most common antiarrhythmic agents. Continuous electrocardiographic recording and analysis proved extremely useful in the management of this case. Mexiletine, a new antiarrhythmic drug, was finally effective in controlling recurrent attacks of ventricular tachycardia.
\end{abstract}

At least 18 viruses are known to affect the heart (Abelmann, 1971). The varicella-zoster virus is one of the most rare. Since the necropsy findings in varicella myocarditis were first described by Hackel (1953), several reports have appeared (Cheatham et al., 1956; Tatter et al., 1964; Morales et al., 1971) mainly concerned with histological changes in cardiac muscle. Recovery from varicella myocarditis has been reported in only 3 patients (De Medeiros Neto et al., 1961; Moore et al., 1969).

This report describes a child who presented with syncopal episodes caused by self-terminating ventricular arrhythmias during the course of clinical varicella. These arrhythmias, in the absence of other recognisable precipitating factors, were thought to be the result of myocarditis occurring as a complication of varicella.

\section{Case report}

A healthy 10-year-old boy became ill after contact with his grandfather who had herpes zoster 2 weeks previously. He developed a mild fever followed by a papulo-vesicular eruption on his trunk and face. A diagnosis of varicella was made. Five days later his condition deteriorated and he developed vomiting and headache. At this stage he had 2 syncopal episodes lasting approximately 3 to 4 minutes each. These were associated with eye

IPresent address: Cardiology Department, Killingbeck Hospital Leeds LS14 6UQ.

2Present address: Cardiology Department, Newcastle General, Hospital, Newcastle NE4 6BE. rolling and convulsive movements of his limbs. There was no incontinence or postictal headache and weakness. His general practitioner referred him to hospital with a tentative diagnosis of varicella encephalitis.

His previous medical history was unremarkable except for a febrile convulsion at the age of 3 during an attack of measles.

On admission he was fully conscious, alert, and apyrexial. On his trunk there were fading varicella lesions. His pulse was noted to be irregular, with a rate of 50 to 60 beats per minute. No other abnormal findings were detected in the cardiorespiratory or alimentary systems. Central nervous system examination was normal. A lumbar puncture was performed and showed no increase in cells and a normal protein and sugar content. A further syncopal episode occurred 12 hours after admission. His chest $x$-ray film, full blood count, and electroencephalogram were normal. An electrocardiogram, however, showed a basic sinus bradycardia with frequent multiform ventricular ectopic beats and short bursts of ventricular tachycardia.

Bedside electrocardiographic monitoring and continuous electrocardiographic tape recordings were instituted and these showed that the syncopal episodes were the result of self-terminating runs of ventricular fibrillation (Fig. 1).

Intravenous lignocaine was started but over the following 48 hours several further episodes of ventricular fibrillation occurred, some lasting 1 to $1 \frac{1}{2}$ minutes. Lignocaine, procainamide, and phenytoin proved ineffective in controlling the arrhythmia and he was subsequently transferred to a coronary 
care unit. Continuous electrocardiographic tape recordings were made for the next 8 weeks. The tapes were processed using a Neilson arrhythmia computer and were also visually analysed. This provided a total ventricular ectopic beat count and a continuous graph of ventricular ectopic beat activity for each 24 hours, thus allowing accurate assessment of the effectiveness of further treatment. Surveillance was maintained during a period of mobility with an Oxford Instruments Miniature analogue tape recorder.

On transfer to the coronary care unit treatment

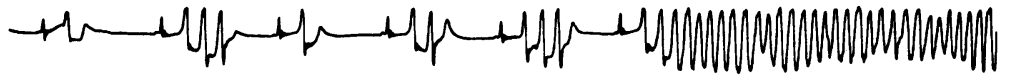

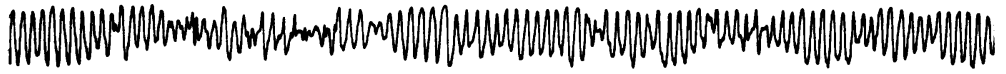

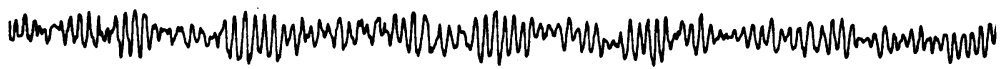

Fig. 1 A write-out from the continuous electrocardiographic tape recording illustrates ventricular tachycardia

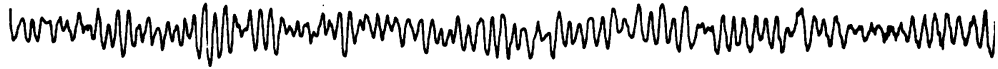
degenerating into ventricular fibrillation. Return to sinus

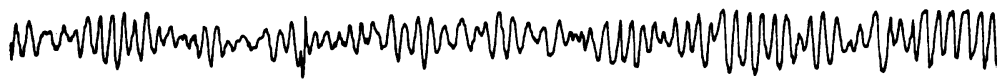
rhythm occurred spontaneously.

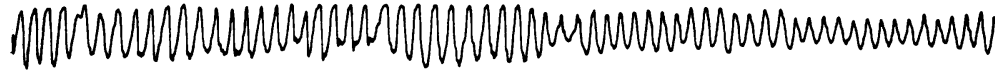
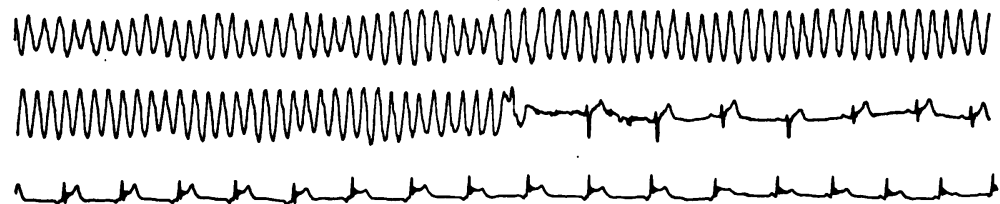

PROPRANOLOL

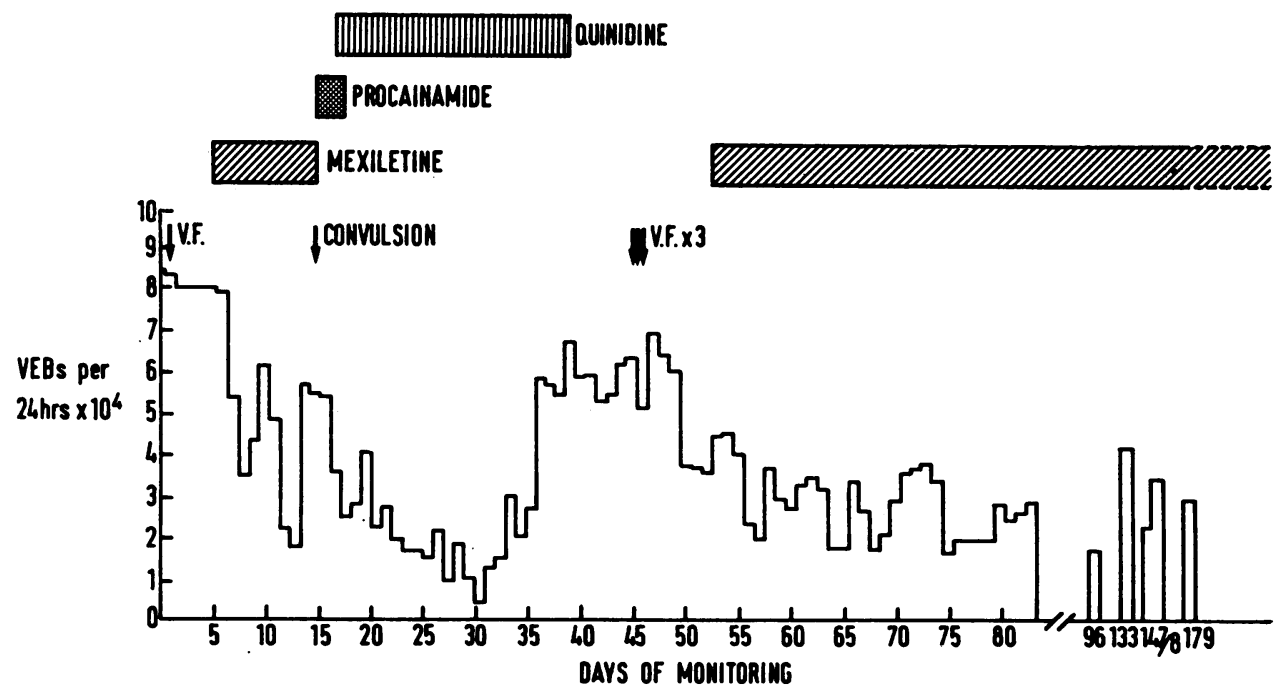

Fig. 2 Histogram of the total ventricular ectopic beat count over the period of observation and treatment. The ventricular ectopic beat count was charted on a daily basis, and the variation in the pattern of the ectopic count in relation to the different treatment schedules is shown. Out-patient ectopic beat counts between the 96th day and the 179 th day are shown on the right. 
was started with mexiletine, a new oral antiarrhythmic agent structurally similar to lignocaine, which has proved effective in ventricular arrhythmias associated with myocardial infarction (Campbell et al., 1975). On a dosage regimen which produced a drug serum concentration at the upper limit of the therapeutic range there was a brief selfterminating convulsive episode not associated with any cardiac arrhythmia. After this mexiletine was discontinued and quinidine started in a dosage of $200 \mathrm{mg} 6$ hourly; this induced an 'L-E syndrome' with fever, lymphadenopathy, hepatosplenomegaly, and a weakly positive antinuclear factor. When this was withdrawn 3 episodes of ventricular fibrillation occurred requiring DC cardioversion. Propranolol was then started without effect on ectopic beat activity. At this time echocardiography, cardiac catheterisation, and angiography were performed and all were within normal limits, apart from reduced contractility of both ventricles which could have been related to the propranolol administration. Atrial pacing at 120 beats per minute abolished the ventricular actopic activity. An endomyocardial biopsy specimen proved too traumatised for histological examination. He was restarted on mexiletine and the propranolol was continued for a further 3 weeks. Serological evidence of varicella infection was obtained 8 weeks after admission with a titre of $1: 128$. Fig. 2 illustrates the total ventricular ectopic count over the period of observation, charted on a daily basis along with the various treatment schedules. In general, lower total ventricular ectopic counts were associated with fewer episodes of ventricular tachycardia. The ectopic activity eventually settled and became stable on mexiletine in a dosage of $450 \mathrm{mg}$ daily.

$\mathrm{He}$ was discharged home on mexiletine 12 weeks after admission, having been free of syncopal episodes for 6 weeks. He remains well at follow-up with stabilisation of ectopic activity as shown by 24-hour electrocardiograms taken at out-patient visits up to 6 months after his illness.

\section{Discussion}

The true incidence of myocardial involvement in the common viral illnesses of childhood is difficult to assess. The majority of cases are subclinical or are found to have minor ' $T$ ' wave changes on the electrocardiogram (Lancet, 1974). Recently myalgia has been reported as being an important diagnostic clue to the presence of myocarditis in viral illness (Lewes et al., 1974) but was absent in this case.

Previous reports of varicella myocarditis have mainly concerned cases of fulminating infection with multiorgan involvement (Hackel, 1953; Tatter et al., 1964; Morales et al., 1971). This case, however, did not show severe infection, and apart from the arrhythmias was associated with a normal clinical course. Documented arrhythmias in varicella myocarditis have been supraventricular in origin (De Medeiros Neto et al., 1961; Tatter et al., 1964; Moore et al., 1969) and resulted in congestive cardiac failure. One report of sudden death in varicella myocarditis showed viral involvement of the conducting tissue at necropsy (Morales et al., 1971). Neither supraventricular arrhythmias nor conduction disturbances were noted in this case. This report, to our knowledge, is the only case of varicella myocarditis recorded in which ventricular arrhythmias have been documented, and suggests a possible mechanism of sudden death in patients with myocardial involvement during the course of a viral illness.

The ventricular arrhythmias recorded in this case were of interest as similarly appearing ventricular arrhythmias known as 'torsade de pointes' ('wave bursts') have been described (Dessertenne, 1966; Motte et al., 1970; Brochier et al., 1972) in which the basic sinus rate is slow with a long QT interval and is interrupted by frequent bursts of bizarrely appearing ventricular tachycardia with altering waveform. The bursts of tachycardia begin with a ' $R$ on $T$ ' ventricular ectopic beat and are usually self-terminating but can degenerate into fatal ventricular fibrillation. These arrhythmias have been described in association with chronic atrioventricular block, quinidine, and chlorpromazine toxicity, the long QT interval syndromes, myocardial ischaemia, magnesium deficiency, potassium depletion (Brochier et al., 1972), and excessive sodium bicarbonate ingestion (Caponnetto et al., 1974). As in this patient the arrhythmias have been difficult to treat and have been resistant to the commonly used antiarrhythmic agents, responding only to rapid pacing. Myocarditis has not previously been reported to produce the 'torsade de pointes' arrhythmia. Impairment of sinoatrial node function attributable to the myocarditis may have produced the sinus bradycardia in this patient. This rhythm in the setting of an already irritable myocardium with frequent ectopic activity almost certainly allowed the development of 'torsade de pointes'. The gradual reduction in ectopic activity may have been the result of a spontaneous improvement resulting from the natural history of the illness and/or more adequate administration of mexiletine as reflected in serum levels which were more consistently within a therapeutic range.

We are grateful to Dr. George Sangster for referring this case. 


\section{References}

Abelmann, W. (1971). Virus and the heart. Circulation, 44, 950-956.

Brochier, M., Motté, G., and Fauchier, J. P. (1972). Tachycardie ventriculaire en torsades de pointe. Actualités Cardio-Vasculaires Médico-Chirurgicales, 6, 171-177.

Campbell, R. W. F., Talbot, R. G., Dolder, M. A., Murray, A., Prescott, L. F., and Julian, D. G. (1975). Comparison of procainamide and mexiletine in prevention of ventricular arrhythmias after acute myocardial infarction. Lancet, 1, 1257-1259.

Caponnetto, S., Pastorini, C., and Masperone, M. A. (1974). Crisi di fibrillazione ventricolare a 'torsione di punta' per introduzione di cospicua quantita' di bicarbonato di sodio. Giornale Italiano di Cardiologia, 4, 211-216.

Cheatham, W. J., Weller, T. H., Dolan, T. F., and Dowei, J. C. (1956). Varicella: report of two fatal cases with necropsy, virus isolation and serologic studies. American Fournal of Pathology, 32, 1015-1028.

De Medeiros Neto, G. A., De Almeida, D. B., and Facchini, F. P. (1961). Miocardite em associacão com varicela. Revista do Hospital das Clinicas de Faculdade de Medicina da Universidade de Sao Paulo, 16, 427-430.

Dessertenne, F. (1966). La tachycardie ventriculaire à deux foyers opposés variables. Archives des Maladies du Coeur et des Vaisseaux, 59, 263-272.
Hackel, D. B. (1953). Myocarditis in association with varicella American fournal of Pathology, 29, 369-379.

Lancet (1974). Viruses and heart disease. 2, 991.

Lewes, D., Rainford, D. J., and Lane, W. F. (1974). Symptomless myocarditis and myalgia in viral and myocoplasma pneumoniae infections. British Heart fournal, 36, 924-932.

Moore, C. M., Henry, J., Benzing, G., and Kaplan, S. (1969). Varicella myocarditis. American fournal of Diseases of Children, 118, 899-902.

Morales, A. R., Adelman, S., and Fine, G. (1971). Varicella myocarditis: a case of sudden death. Archives of Pathology, 91, 29-31.

Motte, G., Coumel, P. N., Abitbol, G., Dessertenne, F., and Slama, R. (1970). Le syndrome QT long et syncopes par 'torsades de pointe'. Archives des Maladies du Coeur et des Vaisseaux, 63, 831-853.

Tatter, D., Gerard, P. W., Silverman, A. H., Wang, C., and Pearson, H. E. (1964). Fatal varicella pancarditis in a child. American Fournal of Diseases of Children, 108, 88-93.

Requests for reprints to Dr. Garrick I. Fiddler, Killingbeck Hospital, Leeds LS14_6UQ. 